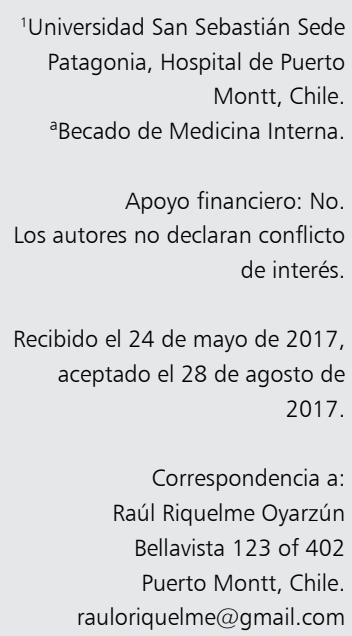

\section{Cambios en la presentación clínica de la influenza A H1N1pdm09 después de la pandemia}

\author{
RAÚL RIQUELME ${ }^{1}$, MARÍA LUISA RIOSECO ${ }^{1}$, \\ KAREN VELÁSQUEZ ${ }^{1, a}$, FERNANDO CAMPOS ${ }^{1, a}$, \\ BENJAMÍN GAYÁN ${ }^{1, a}$, CRISTIAN MEDINA ${ }^{1}$, \\ CARLOS INZUNZA ${ }^{1}$, MAURICIO RIQUELME ${ }^{1}$
}

\section{Changes in the clinical presentation of Influenza A H1N1pdm09 after the pandemic}

Background: After the 2009 influenza pandemic the H1N1pdm09 strain circulate seasonally. In 2015, Puerto Montt Hospital in Chile faced a simultaneous outbreak of both seasonal H3N2 and H1N1pdm09 influenza A (IA). Aim: To evaluate the clinical differences between the two viral strains and recent changes in the behavior of H1N1pdm09 IA. Material and Methods: We set up a retrospective study including every adult hospitalized in Puerto Montt Hospital in 2015 due to IA, confirmed by reverse transcription polymerase chain reaction. We compared epidemiological data, clinical presentation, complications, and the outcome of patients with H1N1pdm09 versus those with seasonal influenza. In parallel, we compared 62 cases of thatH1N1 IA from 2015 with 100 cases who were hospitalized and analyzed in 2009. Results: Between July and October 2015, 119 adults with confirmed IA were hospitalized. From 2009 to 2015, the mean age of patients with IAH1N1pdm09 increased from $40.4 \pm 17$ to $58.8 \pm$ 16 years $(p<0.01)$. Pneumonia as the cause of hospitalization decreased from 75 to $58 \%$ of patients, $(p=0.04)$. Likewise, the presence of comorbidities increased from 53 to $74 \%,(p<0.01)$. Compared with seasonal H3N2, patients with IAH1N1pdm09 IA were more likely to require intensive care $(p<0.01)$ and mechanical ventilation $(p<0.01)$ and developed septic shock $(p=0.03)$. Their mortality was non-significantly higher (13 and 5\% respectively). Conclusions: The clinical presentation of H1N1pdm09 IA has varied over time and now affects an older population, with a greater number of comorbidities. It also appears to be adopting the clinical behavior of a classic seasonal influenza virus.

(Rev Med Chile 2017; 145: 980-986)

Key words: Chile; Influenza A Virus; Influenza A Virus, H1N1 Subtype; Pneumonia, Viral.
L a influenza estacional epidémica se caracteriza por brotes generalmente invernales que afectan más gravemente a menores de $5 \mathrm{y}$ a mayores de 50 años de edad. Desde 1977, los virus influenza A (IA) H3N2, H1N1 y virus influenza $B$ (IB) han co-circulado provocando brotes estacionales de mayor o menor magnitud asociados a una mayor mortalidad en ancianos. Durante el año 2009, apareció un nuevo virus IA H1 que se expandió rápidamente determinando que la OMS elevara precozmente el nivel de alerta al de una pandemia ${ }^{1}$. Este virus H1N1pdm09 mostró un comportamiento clínico diferente al estacional H3N2, afectando especialmente a personas más jó- 
venes, con menos comorbilidades y produciendo un cuadro de mayor extensión radiológica, compromiso respiratorio agudo, necesidad de ingreso a UCI y de ventilación mecánica $(\mathrm{VM})^{2,3}$. En los años posteriores a la pandemia se ha observado que la cepa H1N1pdm09 ha circulado en forma estacional con variaciones regionales ${ }^{4}$.

El Hospital de Puerto Montt (HPM), hospital general de 500 camas, es centro de referencia de la región administrativa de Los Lagos, sur de Chile. En el año 2015, el HPM se vio enfrentado a un brote simultáneo de H1N1pdm09 y H3N2 y tuvimos la oportunidad de comparar las diferencias clínicas entre ambas cepas en los pacientes adultos que se hospitalizaron, especialmente los que cursaron con neumonía. Así mismo, quisimos ver eventuales diferencias entre aquellos con neumonía por H1N1pdm09 respecto a lo casos que vimos en nuestro Hospital el año 2009 por esta misma cepa ${ }^{5}$.

\section{Material y Método}

Se revisaron los registros de microbiología del Laboratorio Clínico del HPM buscando todos los casos con IA demostrada por RT-PCR (del inglés, Reverse Transcription-Polymerase Chain Reaction) y se realizó un estudio retrospectivo que incluyó a todos los adultos que fueron hospitalizados durante el año 2015 por un cuadro de influenza. En nuestro hospital, el virus influenza se estudió en muestras de aspirado nasofaríngeo y en primera instancia, se realizó una inmunofluorescencia directa (IFD) para virus influenza A y B; las muestras negativas por IFD fueron analizadas mediante RT-PCR para ambos virus. Durante la pandemia del 2009, la tipificación del IA se realizaba en el Instituto de Salud Pública (ISP) y a partir del año 2010, la tipificación se realizó localmente mediante RT-PCR. Se consignaron antecedentes epidemiológicos, clínicos y radiológicos, incluyendo edad, sexo, comorbilidades, hábitos de alcohol y tabaco, uso de antibióticos previos y oseltamivir. Para objetivar si habían recibido la vacuna anti-influenza (VAI) del año se utilizó la base de datos del Registro Nacional de Inmunizaciones (RNI). Se consideró que un paciente tenía hiperreactividad inducida por IA si presentaba evidencia de broncoespasmo en ausencia de historia previa de asma o hiperreactividad. Se determinó que había una neumonía adquirida en la comunidad (NAC) si había un infiltrado nuevo en la radiografía de tórax sin una clara alternativa diagnóstica. Todos los pacientes fueron caracterizados clínicamente al momento del ingreso al HPM. El patrón radiológico fue clasificado como alveolar, intersticial o mixto y se registró la presencia de derrame pleural. La extensión radiológica se estimó de acuerdo al número de lóbulos afectados y al compromiso bilateral. La causa de la hospitalización se atribuyó a neumonía, descompensación de comorbilidades u otra. En aquellos que ingresaron a UPC se consignó la presencia de shock, falla respiratoria, SDRA, necesidad de VM y falla renal aguda. Se identificaron los factores asociados con mortalidad en el total de los pacientes incluidos en el estudio. Se compararon las diferencias del huésped, presentación clínica y pronóstico de los casos asociados a cada cepa de IA. Para efectos del análisis se excluyeron las formas de etiología mixta.

Para evaluar eventuales cambios en la presentación de la IAH1N1pdm se confeccionó una base de datos que incluyó 100 casos hospitalizados en el HPM durante la pandemia de 2009, con resultados previamente publicados ${ }^{5}$ y los casos ingresados el 2015.

Análisis estadístico: Se usó el paquete estadístico SPSS 21 para Windows y los resultados se expresan como promedio \pm DS. Las variables continuas fueron comparadas mediante la prueba $t$ de Student y las categóricas con test de chi cuadrado o de Fisher cuando fue apropiado. Para buscar factores independientes asociados a mortalidad se realizó un análisis multivariado de los datos mediante regresión logística. Un valor bilateral de $\mathrm{p}<0,05$ se consideró significativo.

\section{Resultados}

Durante el año 2015 ingresaron al HPM 128 adultos con IA demostrada por RT-PCR. Se excluyeron del análisis 9 casos: 3 con IB, 2 con H1N1pdm09+H3N2, uno con H3N2+IB y 3 casos asociados a bacteriemia por Streptococcus pneumoniae. De esta forma se incluyeron en el estudio 119 casos demostrados de IA. Las principales características de este grupo pueden verse en la Tabla 1. En el RNI estaban registrados el 75\% $(\mathrm{n}=89)$ de los casos y de ellos, habían recibido VAI el 50\% ( $\mathrm{n}=44)$; la edad de los vacunados 
Tabla 1. Características de pacientes con IA ingresados al Hospital de Puerto Montt el año 2015 y comparación de IAH1N1 pdm09 con IA estacional H3N2

\begin{tabular}{|c|c|c|c|c|}
\hline & $\begin{array}{c}\text { Total casos IA } \\
n=119 \\
n(\%)\end{array}$ & $\begin{array}{c}\text { IA H1N1pdm } \\
n=62 \\
n(\%)\end{array}$ & $\begin{array}{c}\text { IA H3N2 } \\
n=57 \\
n \text { (\%) }\end{array}$ & $\mathbf{p}$ \\
\hline Edad (años \pm DS) & $64 \pm 17$ & $59 \pm 16$ & $70 \pm 17$ & $<0,001$ \\
\hline Edad $\geq 65$ años & $63(53)$ & $23(37)$ & $40(70)$ & $<0,001$ \\
\hline $\begin{array}{c}\text { Comprobación diagnóstica } \\
\text { - IFD (-) / RT-PCR (+) } \\
\text { - IFD (+) / RT-PCR (+) }\end{array}$ & $\begin{array}{l}68(57) \\
51(43)\end{array}$ & & & \\
\hline Sexo masculino & $55(46)$ & $35(56)$ & $20(35)$ & 0,027 \\
\hline Días hospitalización $n \pm$ DS & $11,7 \pm 14$ & $13 \pm 16$ & $10 \pm 11$ & 0,378 \\
\hline Vacuna anti influenza & $44(37)$ & $15(34)$ & $29(64)$ & 0,006 \\
\hline$\geq 1$ comorbilidad & $89(75)$ & $48(77)$ & $41(72)$ & 0,531 \\
\hline $\begin{array}{l}\text { - Enfermedad pulmonar crónica } \\
\text { - Cardiopatía } \\
\text { - Diabetes } \\
\text { - Desorden neurológico } \\
\text { - Insuficiencia renal crónica } \\
\text { - Hepatopatía crónica } \\
\text { - Obesidad } \\
\text { - Alcoholismo } \\
\text { - Embarazo }\end{array}$ & $\begin{aligned} 40 & (37) \\
32 & (27) \\
29 & (25) \\
18 & (15) \\
18 & (15) \\
7 & (6) \\
7 & (6) \\
4 & (3) \\
3 & (2,5)\end{aligned}$ & $\begin{aligned} 23 & (37) \\
16 & (26) \\
19 & (31) \\
10 & (16) \\
9 & (15) \\
4 & (6) \\
4 & (6) \\
3 & (5) \\
1 & (2)\end{aligned}$ & $\begin{array}{ll}17 & (30) \\
16 & (28) \\
10 & (18) \\
8 & (14) \\
9 & (16) \\
3 & (5) \\
3 & (5) \\
1 & (2) \\
2 & (4)\end{array}$ & $\begin{array}{l}0,442 \\
0,838 \\
0,134 \\
0,802 \\
1,000 \\
1,000 \\
1,000 \\
0,620 \\
0,606\end{array}$ \\
\hline Hiperreactividad bronquial por virus & $12(10)$ & $8(13)$ & $4(7)$ & 0,368 \\
\hline Neumonía & $54(45)$ & $36(58)$ & $18(32)$ & 0,006 \\
\hline - Edad de los con neumonía & $62 \pm 17$ & $58 \pm 16$ & $70 \pm 17$ & 0,019 \\
\hline Ingreso a UPC & $27(23)$ & $22(35)$ & $5 \quad(9)$ & 0,001 \\
\hline VMI & $16(13)$ & $14(63)$ & $2(4)$ & 0,003 \\
\hline Shock séptico & $6(5)$ & $6(10)$ & 0 & 0,028 \\
\hline Fallecidos & 11 (9) & $8(13)$ & 3 (5) & 0,209 \\
\hline
\end{tabular}

era mayor que la de los no vacunados $(71 \pm 15$ vs $60,5 \pm 17 ; \mathrm{p}$ 0,003). Todos los casos ocurrieron entre los meses de julio y octubre y el $87 \%$ en los meses de agosto y septiembre. Hubo 54 casos (43\%) compatibles con neumonía y en ellos la Rx tórax al ingreso mostró compromiso bilateral en el 69\% (37/54) y fue mayor de 3 lóbulos en 50\% (27/54). En aquellos sin neumonía, la Rx tórax fue normal al ingreso en $65 \%(42 / 65)$ y el resto tenía imágenes compatibles con cardiopatía o enfermedad pulmonar no infecciosa (secuelas, enfisema, enfermedad pulmonar difusa). Los principales motivos de hospitalización fueron NAC y comorbilidades, cada una con 50 casos (42\%). Doce pacientes se hospitalizaron por bronquitis aguda con fiebre. El 90\% recibió antibióticos empíricos preferentemente ceftriaxona en monoterapia (40\%) o asociada con quinolona o macrólido. Sólo 2 pacientes habían recibido oseltamivir antes de ingresar y durante la hospitalización se le indicó a $98 \%$ de los casos. Veintinueve casos cumplían con criterios de neumonía grave para ingresar a UPC ${ }^{6}$ e ingresaron 21, requiriendo VM 14. La limitación del esfuerzo terapéutico $(n=7)$ y falta de cupo en la red $(\mathrm{n}=1)$ explican que 8 pacientes no ingresaran a UPC. Fallecieron 11 pacientes $(9,2 \%)$ y 5 de ellos estaban vacunados (3 H1N1pdm09 y 2 H3N2). La edad de los fallecidos fue de 66,3 $\pm 19,4$ años y la mortalidad fue atribuible a la neumonía en $90 \%(10 / 11)$ y en un caso se asoció con comor- 
bilidades múltiples descompensadas. Los factores de riesgo para fallecer asociados a influenza $A$ pueden verse en la Tabla 2. Excepto la neumonía, ninguna morbilidad ni signo clínico se asoció con mortalidad.

Hubo 62 pacientes (52\%) con H1N1pdm09 y $57(48 \%)$ con H3N2 estacional. En la Tabla 1 se muestran las diferencias encontradas entre ambos grupos. En aquellos con neumonía no encontramos diferencias significativas en síntomas, signos físicos, exámenes de laboratorio, tipo de infiltrados, extensión radiológica ni mortalidad.

Las diferencias entre los casos de H1N1pdm09 del año 2015 y del 2009 pueden verse en la Tabla 3.

Tabla 2. Factores asociados a mortalidad por influenza $A$ en 119 casos consecutivos que ingresaron al Hospital de Puerto Montt durante el 2015. Análisis uni y multivariado

\begin{tabular}{|lcccccc|}
\hline & OR & IC 95\% & p & OR & IC 95\% & p \\
\hline Ingreso a UPC & 7,2 & $1,9-27$ & 0,003 & & & \\
\hline PAFI $\leq 200^{*}$ & & & 0,027 & & & \\
\hline Neumonía & 15 & $1,9-123$ & 0.002 & & & \\
VMI** & 10,7 & $1,3-87$ & 0,032 & 10,1 & $1,31-77,6$ & 0,026 \\
\hline Shock séptico & 13 & $1,2-141$ & 0,050 & 5,75 & $1,05-31,6$ & 0,064 \\
\hline SDRA & 13 & & 0,023 & & \\
\hline
\end{tabular}

*Presión arterial de oxígeno/Fracción inspirada de oxígeno, al ingreso al hospital. **Ventilación Mecánica Invasiva.

Tabla 3. Comparación de pacientes hospitalizados por IA H1N1pdm09 los años 2009 y 2015 en el Hospital de Puerto Montt

\begin{tabular}{|c|c|c|c|}
\hline & $\begin{array}{c}\text { IA H1N1pdm } 2009 \\
n=100 \\
n(\%)\end{array}$ & $\begin{array}{c}\text { IA H1N1pdm } 2015 \\
n=62 \\
n(\%)\end{array}$ & \\
\hline Edad (años $\pm D S$ ) & $40,4 \pm 17$ & $58,8 \pm 16$ & $<0,001$ \\
\hline Edad $\geq 65$ años & $11(11)$ & $23(37,1)$ & $<0,001$ \\
\hline Sexo masculino & $47(47)$ & $35(56,5)$ & 0,261 \\
\hline Días hospitalización ( $\mathrm{n} \pm \mathrm{DS}$ ) & $7,9 \pm 8,5$ & $12,8 \pm 16$ & 0,012 \\
\hline Comorbilidades & $53(53)$ & $48(77,4)$ & 0,003 \\
\hline - Enfermedad pulmonar crónica & $28(28)$ & $23(37,1)$ & 0,230 \\
\hline - Cardiopatía & $12(12)$ & $16(25,8)$ & 0,032 \\
\hline - Insuficiencia cardiaca congestiva & 4 (4) & $10(16,1)$ & 0,010 \\
\hline - Diabetes & $16(16)$ & $19(30,6)$ & 0,032 \\
\hline - Desorden neurológico & $3(3)$ & $10(16,1)$ & 0,005 \\
\hline - Insuficiencia renal crónica & $3(3)$ & $9(14,5)$ & 0,011 \\
\hline - Hepatopatía crónica & $2(2)$ & $4(6,5)$ & 0,204 \\
\hline - Obesidad & $11(11)$ & $4(6,5)$ & 0,411 \\
\hline - Cáncer & 1 (1) & $4(6,5)$ & 0,071 \\
\hline - Alcoholismo & $2(2)$ & $3(4,8)$ & 0,372 \\
\hline - Embarazo & 7 (7) & $1(1,6)$ & 0,156 \\
\hline - Compromiso de conciencia & $5(5)$ & $11(17,7)$ & 0,013 \\
\hline Descompensación enfermedad previa & $28(28)$ & $12(54,5)$ & 0,024 \\
\hline Neumonía & $75(75)$ & $36(58,1)$ & 0,036 \\
\hline Ingreso a Unidad Paciente Crítico & $25(25)$ & $22(35,5)$ & 0,160 \\
\hline Fallecen & $9(9)$ & $8(12,9)$ & 0,433 \\
\hline
\end{tabular}


Los últimos eran más jóvenes, tenían menos comorbilidades, cursaron más frecuentemente con neumonía y menor compromiso de conciencia pero no diferían en la indicación de UCI ni en la mortalidad. El rendimiento de la IFD fue de $51 \%$ el 2009 y de $45 \%$ el 2015. No hubo diferencias en los exámenes de laboratorio, infiltrados radiológicos, frecuencia de shock, SDRA o VM. Tampoco se diferenciaron en el uso de antibióticos y antivirales ni en el porcentaje de hiperreactividad inducida por el virus.

\section{Discusión}

El principal hallazgo de nuestro trabajo es evidenciar los cambios en la presentación clínica que ha tenido la IAH1N1pdm desde su aparición el 2009 con un cuadro de mayor agresividad a un comportamiento actual mucho más similar al del clásico virus estacional.

El virus influenza ocasiona todos los años brotes de distinta magnitud, preferentemente invernales, con considerable morbilidad y aunque la mayoría de las veces se manifiesta con cuadros respiratorios auto limitados, hay un grupo de pacientes que cursa con enfermedad severa, complicaciones, hospitalización y mortalidad. Desde hace más de 50 años se han descrito factores de riesgo de complicación de la influenza como son las edades extremas de la vida, ciertas enfermedades crónicas y embarazo $^{3,7}$. Las complicaciones más frecuentes son respiratorias, especialmente neumonía, pero también pueden existir compromiso de otros sistemas y exacerbación de patologías crónicas como asma, EPOC y cardiopatías ${ }^{3,7}$. Aunque la edad es un importante factor de riesgo para desarrollar complicaciones respiratorias bajas, la neumonía viral primaria en ancianos es relativamente poco común fuera de los períodos pandémicos, debido a que un gran porcentaje de esta población tiene inmunidad parcial por vacunaciones $y / o$ infecciones previas ${ }^{8}$. La serie que describimos en nuestro trabajo representa bien a aquella población de IA que requiere hospitalización, la mayoría son ancianos que se complican con neumonía y/o presentan descompensación de sus comorbilidades, desarrollan un cuadro clínico serio en que uno de cada 4 pacientes cumple criterios para ingresar a UPC y fallece 9\% (Tabla 1). Los factores asociados a mortalidad que se encontraron en el análisis estadístico (Tabla 2), confirman que la causa de muerte principal es una falla respiratoria grave asociada a neumonía viral primaria. La asociación con infecciones bacterianas también es una forma de presentación importante ${ }^{9} \mathrm{y}$ en nuestra serie, pese a su exclusión del análisis estadístico, tuvimos 3 pacientes con bacteriemia por S. pneumoniae lo que representa $2,5 \%$. Este porcentaje puede parecer bajo al comparar con otras publicaciones ${ }^{10}$, pero se debe considerar que este trabajo no fue diseñado para estudiar otras etiologías.

Posterior a la pandemia, el virus H1N1pdm09 sustituyó al virus estacional H1N1 que circulaba hasta el 2009 y desde entonces, ha persistido con frecuencia variable según la región y el año ${ }^{11}$, presentando algunos cambios epidemiológicos que discutiremos a continuación.

El año 2009, el nuevo virus influenza H1N1pdm09 causó la primera pandemia del siglo $\mathrm{XXI}^{12}$ y en forma casi inmediata se hizo evidente que existían diferencias importantes respecto a la IA estacional relacionadas con las características del huésped, la presentación clínica y el pronósti$\mathrm{CO}^{3}$. Los pacientes afectados por este nuevo virus eran más jóvenes, tenían mayor posibilidad de tener complicaciones del tracto respiratorio inferior, shock, sepsis, falla orgánica múltiple, necesidad de UCI, VM y mayor posibilidad de fallecer ${ }^{2,3,12}$. La gran mayoría de estos estudios incluían solo pacientes hospitalizados, pero información del período postpandémico inmediato muestra que en la población general de todas las edades, aquellos con neumonía por IAH1N1pdm09 requerían también más hospitalizaciones que los $\mathrm{H} 3 \mathrm{~N} 2{ }^{13}$. Sin embargo, hay reportes donde esta diferencia no es evidente probablemente por la inclusión conjunta de poblaciones pediátricas y adultas, ambulatorias y hospitalizadas ${ }^{14}$, debido a que la mayoría de los afectados globalmente por H1N1pdm son niños que cursan con cuadro leve ambulatorio.

El año 2015 tuvimos la oportunidad de comparar nuevamente el comportamiento de la H1N1pdm09 con la cepa estacional H3N2 en pacientes adultos que requieren hospitalización, debido a que en nuestra región hubo un brote simultáneo de ambas cepas (Tabla 1) y objetivamos que la H1N1pdm09 ya no se comporta en forma tan diferente de la H3N2. Actualmente y de acuerdo a nuestros hallazgos, la H1N1pdm09 sigue afectando a una población significativamente más joven, pero el promedio de edad actual de 
los pacientes es de 59 años, bastante cercano al promedio de edad del grupo con H3N2 (70 años). En cambio, la edad promedio de los afectados en la pandemia era de 39,7 años para el H1N1pdm09 y de 69,6 años para el H3N2². La mayor frecuencia de comorbilidades (daño pulmonar, cardiopatía, desorden neurológico y alcoholismo) en los pacientes con H3N2 del año 2009, no se evidencia $\mathrm{y}$ ambos grupos son semejantes en este aspecto. Tampoco hay diferencias en la necesidad de VMNI. Sin embargo, persisten diferencias significativas en el comportamiento de ambas cepas, similar a lo observado durante la pandemia ${ }^{2}$ y que traducen una mayor agresividad del virus H1N1pdm09: la neumonía como causa de hospitalización, el ingreso a UCI y la necesidad de VMI son todavía más frecuentes en los pacientes afectados por esta cepa; la mortalidad, aunque no alcanzó significación estadística, es también más frecuente (Tabla 1).

La similitud actual del H1N1pdm09 con el H3N2 nos llevó a construir una base de datos para comparar el comportamiento de este virus en la pandemia del 2009 con el comportamiento actual (año 2015), utilizando para ello datos nuestros previamente publicados ${ }^{2,5}$. Los cambios que ha presentado la expresión clínica del H1N1pdm09 quedan más claros al comparar estas series de adultos hospitalizados el $2009(\mathrm{n}=100)$ y el 2015 $(n=62)$ en el mismo centro hospitalario (Tabla 3$)$. Observamos que entre ambos períodos hay diferencias significativas, siendo ahora una población de mayor edad en que la descompensación de una enfermedad previa es el motivo principal de hospitalización $(p=0,024)$. La neumonía en cambio, fue menos frecuentemente el 2015 como causa de ingreso que lo registrado en la pandemia (Tabla 3). Hay también un aumento significativo de la presencia de comorbilidad de $53 \%$ a $77,4 \%$ $(\mathrm{p}=0,003)$, coincidente con la mayor edad de la población afectada. Estos cambios en el comportamiento de la IAH1N1pdm09 ya se vislumbraban en el período postpandémico inmediato reportándose que aquellos pacientes que se hospitalizaban por igual cepa, eran de mayor edad y neumonía que los que fueron admitidos durante la pandemia ${ }^{15}$.

Estos hallazgos parecen señalar que hubo un cambio del huésped del H1N1pdm09, que envejeció y por lo tanto también se transformó en una población con alta frecuencia de enfermedades crónicas concomitantes. Esto podría deberse a que en la pandemia del 2009, el nuevo virus afectó principalmente a una población joven con limitada inmunidad preexistente y por lo tanto con mayor riesgo de complicaciones respiratorias graves. Se puede postular que, como consecuencia de haber sido afectados en forma importante durante la pandemia y tal vez expuestos a inmunizaciones en las campañas de vacunación de los años posteriores, los pacientes más jóvenes han tenido la oportunidad de formar un escudo inmunológico que ha hecho que la enfermedad se traslade a un grupo de mayor edad, grupo que tradicionalmente es afectado con mayor frecuencia debido a la presencia de más comorbilidad e inmunocenescencia ${ }^{8}$. A la luz de esta información nos parece que debería extenderse la vacunación a otros grupos etarios de menor edad ya que en la práctica habitual en nuestro país la indicación sigue estando principalmente ligada a la edad sobre 65 años o a la presencia de comorbilidades. Esto se releva aún más con los datos de nuestra serie donde la vacunación fue significativamente más frecuente en los pacientes de mayor edad y más afectados por $\mathrm{H} 3 \mathrm{~N} 2$ y no en los pacientes con H1N1pdm09, más jóvenes y que presentan cuadros más severos.

Creemos que nuestro estudio aporta al conocimiento del comportamiento actual de la IAH1N1pdm09 que se hospitaliza mostrando que la H1N1pdm09 reemplazó a la H1N1 estacional que circulaba antes del 2009, circula en forma conjunta con la cepa $\mathrm{H} 3 \mathrm{~N} 2$ estacional en el período epidémico anual y su comportamiento clínico se está semejando al comportamiento clásico de los virus estacionales, mostrando menor agresividad y afectando a una población de mayor edad y con más patología asociada.

Agradecimientos: A la Sra. María Teresa Silva por su apoyo en el análisis estadístico.

\section{Referencias}

1. Fineberg H. Pandemic preparedness and response-lessons from the H1N1 Influenza of 2009. N Eng J Med 2014; 370: 1335-42.

2. Riquelme R, Torres A, Rioseco ML, Ewig S, Cillóniz C, Riquelme $\mathrm{M}$ et al. Influenza pneumonia: a comparison between seasonal influenza and H1N1 pandemic. Eur Respir J 2010; 36: 864-9. 
3. Reed C, Chavez S, Pérez A, D’Mello T, Kirley D, Aragon $\mathrm{P}$, et al. Complications among adults hospitalized with Influenza: A comparison of seasonal Influenza and the 2009 H1N1 pandemic. Clin Infect Dis 2014; 59 (2): 16674.

4. Organización Panamericana de la salud. Influenza y otros virus respiratorios bajo vigilancia, 2010-2014. Vigilancia regional de la influenza. http://ais.paho.org/ phip/viz/ed_flu.asp

5. Riquelme R, Riquelme M, Rioseco ML, Inzunza C, Gómez Y, Contreras C, et al. Characteristics of hospitalized patients with 2009 H1N1 influenza in Chile. Eur Respir J 2010 Feb 25.

6. Mandell L, Wunderink R, Anzueto A, Bartlett, Campbell G, Dean N, et al. Infectious Diseases Society of America/ American Thoracic Society Consensus Guidelines on the Management of Community-Acquired Pneumonia in Adults. CID 2007; 44 (Suppl 2): 27-72.

7. Rothberg MB, Haessler SD, Brown RB. Complications of viral influenza. Am J Med 2008; 121: 258-64.

8. Falsey AR. Community-Acquired viral pneumonia. Clin Geriatr Med 2007; 23: 535-52.

9. Louria DE, Blumenfeld HL, Ellis JT, Kilbourne ED, Roger DE. Studies of influenza in the pandemics of 1957-1958. Pulmonary complications of influenza. J Clin Invest 1959; 135-38.

10. Murata Y, Walsh EE, Falsey AR. Pulmonary compli- cations of interpandemic influenza A in hospitalized adults. J Infect Dis 2007; 195: 1029-37.

11. Novel Swine-Origin Influenza A (H1N1) Virus Investigation Team. Emergence of a novel swine-origin influenza A (H1N1) virus in humans. N Engl J Med 2009; 360: 2605-15.

12. Rabagliati R, Siri L, Pérez CM, Labarca J, Ferrés M. Pandemic influenza A (H1N1) 2009: epidemiology, clinical features and differences with seasonalinfluenza in Chile. Rev Chilena Infectol 2011; 28 (6): 546-53.

13. Yang SQ, Qu JX, Wang C, Yu XM, Liu YM, Cao B. Influenza pneumonia among adolescents and adults: a concurrent comparison between influenza A (H1N1) pdm09 and A (H3N2) in the post-pandemic period. Clin Respir J 2014; 8 (2): 185-91.

14. Yang ZF, Zhan YQ, Chen RC, Zhou R, Wang YT, Luo Y, et al. A prospective comparison of the epidemiological and clinical characteristics of pandemic (H1N1) 2009 influenza A virus and seasonal influenza A viruses in Guangzhou, South China in 2009. Jpn J Infect Dis 2012; 65 (3): 208-14.

15. Rahamat-Langendoen JC, Tutuhatunewa ED, Schölvinck EH, Hak E, Koopmans M, Niesters HG, et al. Influenza in the immediate post-pandemic era: a comparison with seasonal and pandemicinfluenza in hospitalized patients. J Clin Virol 2012; 54 (2): 135-40. 